\title{
RELAÇÃO ENTRE GEOMORFOLOGIA FLUVIAL E BIODIVERSIDADE E SUA APLICAÇÃO NO PROCESSO DE AVALIAÇÃO AMBIENTAL
}

\section{THE RELATIONSHIP BETWEEN FLUVIAL MORPHOLOGY AND BIODIVERSITY AND ITS APPLICATION IN THE PROCESS OF ENVIRONMENTAL EVALUATION.}

\author{
Carlos Roberto S. Fontenelle Bizerril ${ }^{1}$ \\ ${ }^{1}$ Universidade do Rio de Janeiro (UNI-RIO), Laboratório de Avaliação Ambiental. Endereço para \\ correspondência: rua Roquete Pinto, nº59, apto. 202, Urca,Rio de Janeiro, RJ, Brasil. CEP: 22291-210.
}

\begin{abstract}
The relationship between the physical environment and the biological diversification of fish communities was studied and the use of fluvial morphology as an indicative of priority areas for conservation was examined. As a case study the upper course of rio Macacu, State of Rio de Janeiro, Brazil was selected. Biotic data were obtained during 4 year of samples, conduced in 13 sample units. Each environment explored were characterized for its area, order, extension, slope and number of tributaries. Two new indices (environmental diversity and environmental turnover ratio) were proposed. To asses the correlation between the physical and biotic matrixes the data were compared using the Mantel test. A strong correlation between the physical variables and biodiversity were observed. An index to measure the ecological relevance of fluvial ecosystems based on the physical attributes was presented.
\end{abstract}

Key words: Biodiversidade, avaliação ambiental, peixes de água doce, sudeste do Brasil, biodiversity, environmental evaluation, freshwater fishes, southeastern Brazil.

\section{INTRODUÇÃO}

Embora a avaliação de impactos tenha sido introduzida como instrumento da política ambiental brasileira em 1981 com a promulgação da lei 6938, somente após a edição da Resolução CONAMA nº01 de 1986, definindo o escopo, as responsabilidades e as diretrizes para a sua implementação, é que a análise de impacto, e outras atividades relacionadas ao processo de avaliação ambiental, passou a ser adotada em todos os Estados do País (BRITO, 1995).

Inegavelmente, a maior contribuição da prática da avaliação de impactos no Brasil foi a introdução do princípio de democratização da gestão ambiental, assegurando mecanismos de participação pública no processo de tomada de decisão quanto à administração dos recursos naturais (BRITO, 1995). Contudo, o processo de avaliação como um todo (incluindo a 
elaboração dos estudos, a avaliação e o licenciamento) embora teoricamente bem concebido, exibe falhas que, em determinadas situações, conduzem à sua ineficácia.

Ainda na fase de elaboração dos estudos de avaliação ambiental, sejam eles estudos de viabilidade, inventários ou EIA/RIMAs, um dos principais obstáculos enfrentados pela equipe multidisciplinar envolvida é o tempo disponível para a execução e conclusão dos trabalhos, tendo em vista que estudos de inventários e de viabilidade raramente excedem 6 meses de duração enquanto os trabalhos relacionados com a avaliação de impactos ambientais possuem, atualmente, duração média de 6-8 meses. Trata-se de um intervalo de tempo extremamente reduzido, especialmente no que tange a esfera dos estudos do meio biótico.

Os estudos de meio biótico inseridos dentro do contexto do processo de avaliação ambiental embora sigam métodos diversos, que variam de acordo com a meta final do tipo de processo envolvido, assemelham-se quanto a um aspecto: em todos há uma preocupação de identificar, dentro das áreas em estudo, quais as subunidades de georreferenciamento que se notabilizam por manter maior diversidade biológica ou, em outras palavras, concentram, em uma dada região, maior quantidade de informação geno-fenotípica. Tais áreas mostram maior relevância ecológica relativa, constituindo sistemas que devem ser delineados prioritariamente como unidades de conservação (JENNINGS \& REGANOLDS, 1991).

Alcançar este objetivo no curto intervalo de tempo disponível é algo bastante complexo, devido a grande variação temporal natural sofrida pela composição qualitativa das comunidades bióticas, aspecto este que determina a necessidade de inúmeras campanhas para que se tenha uma amostra que represente a real diversidade biológica da área em estudo. Logicamente, um estudo efetuado dentro de um prazo total de 6 meses (o que permite, via de regra, uma campanha de 15-20 dias de duração) raramente é capaz de realizar este tipo de inventário.

Uma alternativa para esta situação é buscar, dentro do conhecimento gerado como produto da pesquisa de determinados ramos da biologia, particularmente na área de ecologia e biogeografia, soluções que permitam viabilizar a prática de estudos de avaliação ambiental com qualidade científica dentro do prazo atualmente disponível.

O presente artigo trabalha dentro dessa diretriz, ou seja, objetiva apresentar uma alternativa aplicada ao estudo de ecossistemas fluviais, mais especificamente ao de comunidades de peixes. No caso, a ictiofauna foi selecionada como segmento biótico de bioindicação de relevância ecológica de ecossistemas aquáticos devido ao forte conteúdo sistêmico do grupo. 
A observação de que profissionais que se encontram diretamente envolvidos com processos de captura de organismos aquáticos (i.e., pescadores, coletores profissionais, biólogos de campo) facilmente reconhecem, a partir de uma rápida avaliação geral de ambientes fluviais, quais sistemas irão fornecer maior riqueza de espécies quando da realização da coleta sugere que existam variáveis ambientais que, quando conjugadas, resultam em uma condição que favorece o incremento da biodiversidade.

Este conhecimento acumulado empiricamente encontra-se igualmente documentado em diversos estudos ecológicos. Dentre as abordagens realizadas neste sentido, destacam-se os trabalhos de MCARTHUR \& WILSON (1963, 1967), SIMBERLOF (1974), DIAMNOND \& MAY (1976) e outros que demonstraram a forte relação existente entre área (A) e o número de espécies (S), relação essa usualmente expressa pela função exponencial dada por $S=b \cdot A^{a}$.

A área influencia o número de espécies de diferentes maneiras, tanto sob o aspecto trófico, visto que ambientes com maiores dimensões gerais viabilizam a ocorrência de espécies com diferentes necessidades espaciais para forageamento, bem como reduzem a intensidade de interações competitivas e de predação (SIMBERLOF \& WILSON, 1969, 1970), como no que diz respeito a interações entre organismos e disponibilidade de nichos potencialmente utilizáveis (COLINVAUX, 1986). No segundo caso, verifica-se que regiões com áreas elevadas são usualmente dotadas de maior diversidade de ambientes ( $\beta$ diversidade) e maior complexidade ambiental quando comparadas com regiões com pequenas áreas. Assim sendo, diversidade de ambientes e complexidade ambiental encontram-se positivamente correlacionadas com o número de espécies (RICKLEFS \& SCHLUTER, 1993).

No caso específico de sistemas fluviais, soma-se a estas três variáveis (área, diversidade de ambientes e complexidade ambiental), a típica zonação exibida pelas comunidades ícticas ao longo de gradientes longitudinais. Esta zonação se expressa na formação de comunidades aquáticas características de cada seção do curso fluvial, taxocenoses estas marcadas pela presença de tipos convergentes de adaptação morfológica (isocenoses, sensu ILLIES, 1962, apud SCHAFER, 1985). Assim sendo, é esperado encontrar maior número de espécies em rios que exibam maior número de seções ecológicas ao longo do gradiente hidrodinâmico.

No presente artigo, a relação entre ambiente físico e biodiversidade é analisada. Para tanto, é proposto um método de caracterização de ambientes fluviais adequado ao estudo da ictiofauna e testada a relação entre cada variável e a diversidade biológica das comunidades ícticas objetivando verificar a aplicabilidade dos conceitos biogeográficos e da geomorfologia 
fluvial na identificação de áreas com maior biodiversidade relativa. Como estudo de caso, foi selecionado o alto curso da bacia do rio Macacu, RJ, Brasil.

\section{MATERIAIS E MÉTODOS}

O local selecionado para a realização do presente estudo foi rio Macacu, um sistema situado no Estado do Rio de Janeiro entre as coordenadas $22^{\circ} 21^{\prime}$ e $23^{\circ}$ de latitude sul e $42^{\circ} 33^{\prime}$ e $43^{\circ}$ de longitude oeste. Consiste em um rio de serra, com forte declividade em seu curso superior antecedendo uma ampla planície fluvial (Figura 1) que conduz à Baía da Guanabara. Sua extensão total é de aproximadamente $72 \mathrm{~km}$, atravessando diversas cidades dos municípios de Cachoeira de Macacu e Magé.

A bacia do Macacu possui área de $1750 \mathrm{~km}^{2}$, sendo os rios Guapi-Açu, Duas Barras, das Covas, Batatal de Baixo, Japuíba, da Pedras e Imbuí seus principais afluentes. A qualidade da água desse sistema fluvial foi classificada como não contaminada, no alto curso, e moderadamente contaminada, com ocorrência de bactérias indicadoras de poluição orgânica, nas baixadas (FEEMA, 1979).

O rio Macacu se destaca, dentre os rios que drenam para a Baía da Guanabara, por

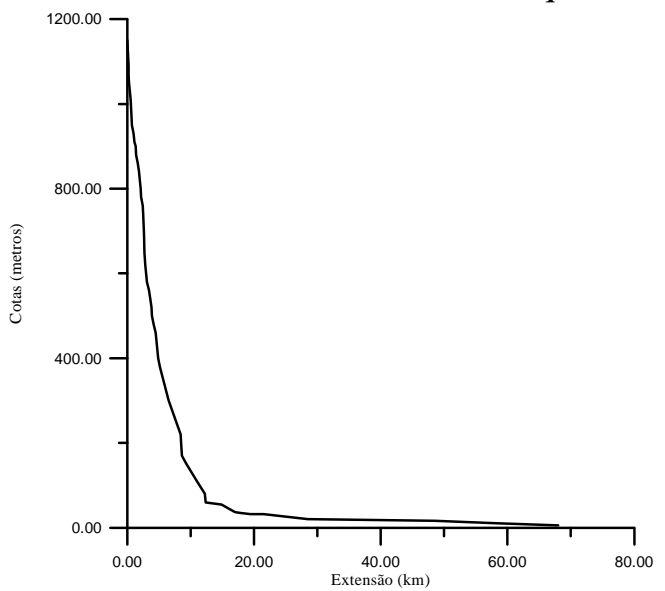

Figura 1 - Perfil longitudinal do rio Macacu, RJ, Brasil

$\begin{array}{lr}\begin{array}{lr}\text { apresentar } \\ \text { periódicas } \\ \text { (LAMEGO, }\end{array} & \text { 1945), } \\ \text { fato este } & \text { que } \\ \text { incentivou } & \text { a } \\ \text { realização } & \text { de } \\ \text { diversas obras } & \text { para } \\ \text { minimizar } & \text { os } \\ \text { impactos causados } \\ \text { pelas inundações. }\end{array}$

$\mathrm{O}$ alto curso do rio

Macacu, RJ, alvo do presente estudo, situa-se entre as coordenadas $22^{\circ} 21^{\prime}$ e $22^{\circ} 30^{\prime}$ de latitude sul e $42^{\circ} 33^{\prime}$ e $42^{\circ} 45^{\prime}$ de longitude oeste. Distingue-se nitidamente das áreas adjacentes, onde predominam alvéolos amplos e formações em meia laranja, pela maior presença de afloramentos cristalinos, com mantos intemperizados e pela menor representatividade de 
rampas de coluviação (ARGENTO, 1985). Este setor reúne um total de 27 sub-bacias, muitas das quais com pequenas dimensões gerais. Destacam-se, por seus maiores portes, os sistemas dos rios Valério, Boa Vista e das Covas.

A cobertura vegetal nativa era de floresta ombrófila densa nas regiões interiores e de manguezais nas áreas de desembocadura. Ambas as formações encontram-se atualmente descaracterizadas, em especial no que diz respeito às fisionomias de floresta ombrófila densa, cujos remanescentes mais expressivos ocorrem apenas nas áreas de cotas mais elevadas e com relevo mais acidentado.

O trabalho em questão foi elaborado em duas etapas abrangendo, em um primeiro momento, aspectos bióticos e, em seguida, parâmetros físicos.

Inicialmente objetivou-se listar a real biodiversidade de ambientes fluviais integrados ao alto curso do rio Macacu. Para tanto, foram realizadas coletas entre janeiro/91 e janeiro/95), totalizando 156 campanhas de campo. As amostragens foram efetuadas em 12 pontos, demarcados no rio Macacu e em 4 subbacias (córregos Colibri, Esgoto, Valona e Valério) (Figura 3). Como instrumentos de captura foram utilizadas peneiras (tela de 0,3 $\mathrm{mm}$ ), tarrafas (malhas de $0,5$ e de $15 \mathrm{~mm})$ e rede de arrasto manual (malha de $1,0 \mathrm{~mm})$.

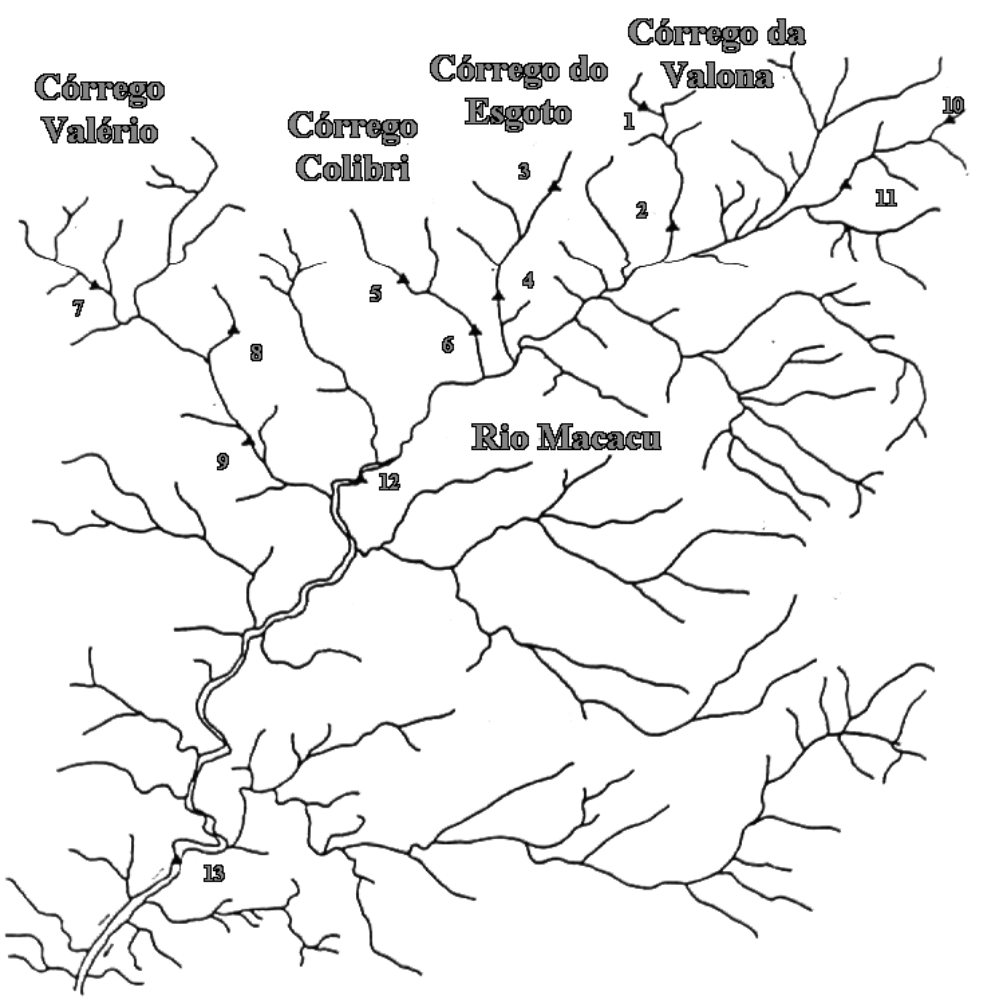

Todo o material coletado foi fixado em campo com formol a $10 \%$

Figura 2 - Alto curso do rio Macacu, RJ, com a indicação dos principais afluentes, e das áreas de amostragem.

e vem sendo 
gradualmente depositado na coleção ictiológica do Museu Nacional do Rio de Janeiro (MNRJ). A identificação das espécies foi realizada com auxílio das chaves elaboradas por BIZERRIL (1994).

Com vistas a identificar associação de espécies e, desta forma, evidenciar padrões biogeográficos existentes dentro das ictiocenoses do alto curso foi utilizado o índice de percentual de dissimilaridade (LUDWIG \& REYNOLDS, 1985) para traduzir numericamente a similaridade de distribuição dos táxons nos diferentes locais amostrados.

Para trabalhar com ictiocenoses de ambientes ao invés da presente em pontos, as unidades amostrais foram agrupadas de modo que cada subunidades de análise foi caracterizada pelo seguintes conjuntos de pontos de amostragem: córrego Valona $\{1,2\}$, córrego Esgoto $\{3$, 4\}, córrego Colibri $\{5,6\}$, córrego Valério $\{7,8,9\}$, rio Macacu subunidade 1 (E1) $\{10,11\}$, rio Macacu subunidade 2 (E2) $\{10,11,12\}$, rio Macacu subunidade 3 (E3) $\{10,11,12$, $13\}$.

A caracterização morfométrica de ambientes fluviais pode ser realizada utilizando-se diferentes conjuntos de elementos (ARGENTO, 1985, STRAHLER, 1952 CHRISTOFOLETTI, 1969, CHRISTFOLETTI et al., 1974), selecionados de acordo com o escopo do trabalho. Para o presente estudo a caracterização dos rios e das sub-bacias estudadas foi efetuada considerando variáveis ambientais que expressem aspectos de dimensão geral, complexidade e diversidade ambiental. Assim sendo, foram consideradas as seguintes variáveis:

1. Área - Obtida adotando-se a técnica de pesagem (cf. CHRISTOFOLETTI et al., 1974).

2. Hierarquia fluvial - Dada pelo método de STRAHLER (1952);

3. Extensão do canal principal - Comprimento total do canal principal do rio Macacu em cada subunidade de amostragem e dos canais principais das sub-bacias;

4. Extensão total do sistema - Obtido pelo somatório das extensões de cada elemento que compõem o conjunto de drenagens considerado;

5. Gradiente - Obtido pela relação entre as altitudes máximas $\left(\mathrm{A}_{\max }\right)$ e mínima $\left(\mathrm{A}_{\min }\right)$ (i.e., amplitude) e a extensão do canal principal (L) (Gradiente $\left.=A_{\max }-A_{\min } / L\right)$;

6. Riqueza de tributários - Dada pela contagem dos tributários de cada sub-bacia ou de cada sub-região do rio Macacu.

Além destes aspectos físicos, foram elaboradas mais duas variáveis que objetivam traduzir a diversificação de ambientes identificadas ao longo do canal fluvial principal de uma 
determinada bacia hidrográfica. Estes índices, nomeados Índice de Diversidade Ambiental e Taxa de Alteração Ambiental sintetizam em um valor numérico características de altitude e declividade e são descritos abaixo:

1. Diversidade ambiental - Para obtenção da diversidade de ambientes foi inicialmente traçado o perfil longitudinal de cada sistema estudado, como descrito em CUNHA \& GUERRA (1996). O eixo do comprimento do perfil foi subdivido em intervalos com extensão de 570 metros e cada subárea reconhecida foi caracterizada quanto a declividade e a altitude média $\left(A m\right.$, sendo $\left.A m=A_{\max }+A_{\min } / 2\right)$. Os valores produzidos foram comparados quanto a sua semelhança utilizando $o$ índice de Bray-Curtis (bjk $=\sum\left|X_{i j}-X_{i k}\right| / \sum\left(X_{i j}+X_{i k}\right)$, sendo Xij e Xik os valores da variável X nas subáreas j e k) e os escores finais foram agrupados por UPGMA (SNEATH \& SOKAL, 1973).

Uma vez efetuado este processo foi obtido a extensão de cada grupo ambiental obtido e estes dados foram lançados na equação originalmente proposta por SHANNON (1948) para calcular o conteúdo informativo de processos estocásticos de uma fonte de transmissão constante, a qual atualmente é de amplo uso em estudos ecológicos como um índice de diversidade. $\mathrm{O}$ índice de diversidade $\left(\mathrm{H}^{\prime}\right)$ é dado, em sua versão modificada, utilizando o nat como unidade de informação, por $\mathrm{H}^{\prime}=-\sum_{n=1}^{n} P i$. $\ln P i$ onde, na presente abordagem, $\mathbf{P i}$ é a probabilidade de ocorrência de um determinado ambiente dentro do canal principal.

2. Taxa de alteração ambiental - A taxa de alteração ambiental é um subproduto da análise supracitada e atua como uma informação complementar. Dois sistemas fluviais podem apresentar a mesma diversidade ambiental, contudo a diferença interna entre os ambientes que os compõe pode se dar em patamares distintos. Para calcular este aspecto como uma taxa de alteração ambiental (TA) considerou-se o valor final do agrupamento das sub-áreas delimitadas em cada região de estudo. Assim sendo, considerando um ambiente hipotético no qual o agrupamento de todas as sub-áreas se deu ao nível de 0,4 tem-se TA = 0,4.

Todos os aspectos supracitados foram obtidos a partir da interpretação da carta 1:50.000 de Nova Friburgo, elaborada pelo IBGE. Correlações entre a biodiversidade e as variáveis ambientais foram realizadas utilizando o índice não-paramétrico de Spearman (ZAR, 1984). 
A comparação entre a diversidade biológica (i.e., riqueza de espécies) das comunidades de cada ponto foi efetuada pelo índice de Dice, gerando uma matriz de afinidade biótica. $\mathrm{O}$ índice de Bray-Curtis foi utilizado para produzir a matriz ambiental. Em ambos os casos o agrupamento foi efetuado por UPGMA. Para comparar a matriz ambiental e a matriz biótica e, desta forma, testar a hipótese nula de não interrelação entre as mesmas, foi aplicado o teste de Mantel (Zyx) (25), dado por Zyx = S Xij Yij, onde Xij e Yij são os elementos extra-diagonal das matrizes $\mathrm{X}$ e $\mathrm{Y}$ a serem comparadas. A significância de Zyx foi obtida comparando-se o valor observado com sua distribuição permutacional, dada pela comparação de uma das matrizes com a permutação aleatória dos elementos ij da outra matriz. Para o presente estudo, foram efetuadas 1000 permutações.

\section{RESULTADOS E DISCUSSÃO}

A região do alto curso do rio Macacu possui suas ictiocenoses compostas por 32 espécies, sendo a maioria pertencente a ordem Siluriformes. Esta relação de dominância de Siluriformes sobre os demais táxons constitui um padrão característico da região leste do Brasil (BIZERRIL, 1994, 1995), sendo particularmente acentuado nas áreas de alto curso dos rios, onde a condição de elevado hidrodinamismo favorece a ocupação do local por espécies demersais, um hábito predominante dentre os Siluriformes (BURGESS, 1989). A relação das espécies inventariadas é apresentada na TABELA I.

Quanto a relação entre extinções e colonizações verificadas ao longo do canal fluvial observou-se que não ocorrem perdas de espécies quando da passagem do ambiente situado nas cotas mais elevadas (i.e., nascentes do rio Macacu e dos córregos Valério, Valona, Colibri e Esgoto) para os demais sistemas. Assim, não existem grupos com distribuição restrita à área próxima as nascentes e o processo de alteração espacial no número de espécies como um todo caracteriza-se pela adição de táxons (sensu SHELFORD, 1911).

O agrupamento dos valores de similaridade de distribuição das espécies permitiu reconhecer 6 grupos ecológicos de táxons, representando espécies com distribuição restrita às estações demarcadas no trecho intermediário e final do rio Macacu (grupo 1 - Hoplias malabaricus, Astyanax sp.1, A. taeniatus, Trichomycterus immaculatus, Hypostomus punctatus, Synbranchus marmoratus), táxons amplamente distribuídos no rio, ocorrendo em todas as unidades amostradas (grupo 2 - Characidium vidalii, Rhamdioglanis transfasciatus, 
Trichomycterus sp., Neoplecostomus microps, Schizolecis gunteri, Phalloceros caudimaculatus), espécies presentes apenas no trecho final do rio Macacu (grupo 3 Characidium interruptum, Homodiateus passarellii, Corydoras nattereri), espécies ocorrentes nos trechos intermediários e final do rio Macacu e no córrego Valério (grupo 4 Bryconamericus ornaticeps, Mimagoniates microlepis, Acentronichthys leptos, Rhamdia sp., Microlepidogaster notatus, Parotocinclus maculicauda, Rineloricaria sp.1, Rineloricaria sp.2, Gymnotus pantherinus, Cichlasoma facetum, Awaous tajasica, Imparfinis piperatus), espécies presentes no trecho intermediário e final do rio Macacu e nos córregos Valério e do Esgoto (Ancistrus sp., Corydoras barbatus, Kronichthys heylandi, Geophagus brasiliensis) e espécie coletada no córrego Valério e no trecho final do rio Macacu (grupo 6 - Pimelodella lateristriga).

\section{TABELA I - RELAÇÃO DAS ESPÉCIES DE PEIXES DO ALTO RIO MACACU, RJ, BRASIL.}

\section{CHARACIFORMES \\ ERYTHRINIDAE \\ Hoplias malabaricus (Bloch, 1794) \\ CRENUCHIDAE}

Characidium vidalii Travassos, 1946

C. interruptum (Pellegrin, 1909)

\section{CHARACIDAE}

Astyanax sp. 1

A. taeniatus (Jenyns, 1842)

Bryconamericus ornaticeps Bizerril \& Peres-Neto, 1995

Mimagoniates microlepis (Steindachner, 1876)

\section{PIMELODIDAE}

\section{SILURIFORMES}

Acentronichthys leptos Eigenmann \& Eigenmann, 1889 Imparfinis piperatus Eigenmann \& Norris, 1900 Pimelodella lateristriga (Mueller \& Troschel, 1849) Rhamdioglanis transfasciatus Ihering, 1907 Rhamdia sp.

\section{TRICHOMYCTERIDAE}

Homodiateus passarellii (Ribeiro, 1944)

Trichomycterus sp.

T. immaculatus (Eigenmann \& Eigenmann, 1889)

CALLICHTHYIDAE

Corydoras barbatus (Quoy \& Gaimard, 1824)

C. nattereri Steindachner, 1877

\author{
LORICARIIDAE \\ Ancistrus sp. \\ Hypostomus punctatus Valenciennes, 1840 \\ Microlepidogaster notatus (Eigenmann \& Eigenmann, \\ 1889 \\ Parotocinclus maculicauda (Steindachner, 1877) \\ Neoplecostomus microps (Steindachner, 1876) \\ Rineloricaria sp.1 \\ Rineloricaria $\mathrm{sp} .2$ \\ Kronichthys heylandi (Boulenger, 1900) \\ Schizolecis guntheri Britski \& Garavello, 1984

\section{GYMNOTIDAE}

\section{GYMNOTIFORMES} \\ Gymnotus pantherinus Steindachner, 1876

\section{POECILIIDAE} \\ CYPRINODONTIFORMES \\ Phalloceros caudimaculatus (Hensel, 1868) \\ SYNBRANCHIFORMES

\section{SYNBRANCHIDAE} \\ Synbranchus marmoratus Bloch, 1795

\section{CICHLIDAE} \\ PERCIFORMES \\ Cichlasoma facetum (Jenyns, 1842) \\ Geophagus brasiliensis (Quoy \& Gaimard, 1824) \\ GOBIIDAE \\ Awaous tajasica (Lichstein, 1822)
}


Agrupando as unidades amostrais com base na biodiversidade (i.e., número de espécies) de cada ponto obteve-se o arranjo hierárquico apresentado na Figura 4, no qual se observa a existência de 3 tipos de sistemas, representativos de áreas com elevada biodiversidade (grupo 1 - rio Macacu nos pontos E2 e E3), baixo número de espécies (grupo 2 córregos Esgoto, Valona, Colibri e Macacu, no ponto E1) e com riqueza taxonômica intermediária (grupo 3 - córrego Valério), mais fortemente associada ao complexo 1.

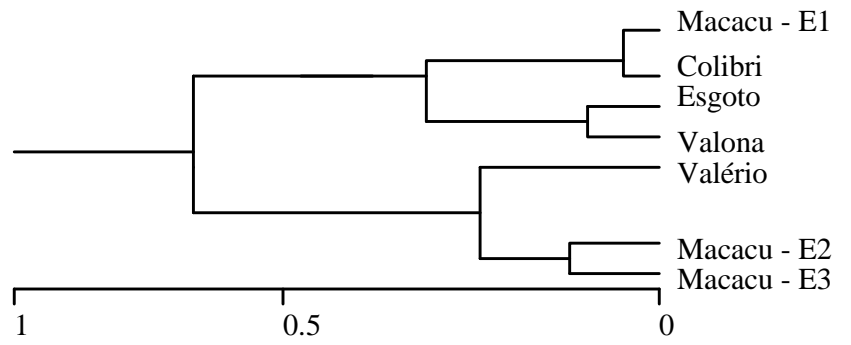

Figura 4 - Agrupamento das unidades amostrais com base na similaridade no número de espécies.

As correlações entre as variáveis fisiográficas e a biodiversidade encontramse na TABELA II. Quanto ao último aspecto, foram obtidos valores elevados de correlação entre todas as variáveis consideradas e o número de espécies (SP), valores estes que, no caso específico da relação entre SP e extensão, extensão total e ordem apresentaram significância estatística. A única correlação negativa entre SP e as variáveis fisiográficas obtida foi para o par SP/Gradiente (G).

TABELA II - CORRELAÇÕES ENTRE AS VARIÁVEIS FÍSICAS E BIÓTICAS.

\begin{tabular}{c|c|c|c|c|c|c|c|c|c}
\hline & $\mathbf{A}$ & $\mathbf{D}$ & $\mathbf{E}$ & $\mathbf{E T}$ & $\mathbf{O}$ & $\mathbf{T A}$ & $\mathbf{R T}$ & $\mathbf{G}$ & SP \\
\hline $\mathbf{A}$ & - & $1^{*}$ & $0.955^{*}$ & 0.8571 & $0.9258^{*}$ & 0.846 & 0.846 & $-0,789$ & 0.818 \\
$\mathbf{D}$ & & - & $0.955^{*}$ & 0.8571 & $0.9258^{*}$ & 0.846 & 0.846 & $-0,789$ & 0.818 \\
$\mathbf{E}$ & & & - & 0.882 & $0.932^{*}$ & $0.936^{*}$ & 0.818 & $-0,998^{*}$ & $0.908^{*}$ \\
$\mathbf{E T}$ & & & & - & $0.925^{*}$ & 0.720 & $0.955^{*}$ & $-0,871$ & $0.927^{*}$ \\
$\mathbf{O}$ & & & & & - & 0.7788 & $0.932^{*}$ & $-0,756$ & $0.903^{*}$ \\
TR & & & & & & - & 0.581 & $-0,888^{*}$ & 0.844 \\
RT & & & & & & & - & $-0,987^{*}$ & 0.816 \\
G & & & & & & & & - & $-0,879$ \\
SP & & & & & & & & & - \\
\hline
\end{tabular}

$\mathrm{A}=$ Área, $\mathrm{D}=$ Diversidade, E = Extensão do canal principal, ET = Extensão Total, $\mathrm{O}=$ Ordem, TA = Taxa de transformação, RT= Riqueza de tributários, $\mathrm{G}=$ Gradiente e $\mathrm{SP}=$ Número de espécies; * - P<0,05

Correlação positiva entre a ordem, a diversidade ambiental e o número de espécies foi igualmente observada por autores como PENZACK \& MANN (1990) e WHITESIDE \& MCNATT (1972), para ambientes da região holártica, e por BIZERRIL (1995), em um rio do leste do Brasil. Os dados ora obtidos reforçam a tendência de covariação no mesmo sentido destas variáveis. 
O agrupamento dos escores de similaridade ambiental calculados na comparação entre

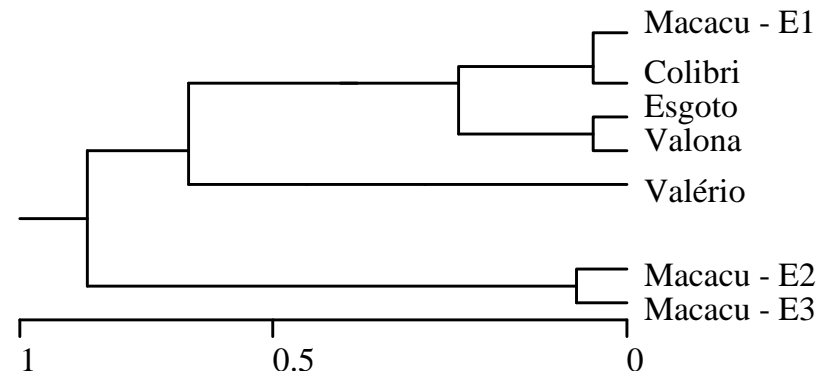

Figura 5 - Agrupamento das unidades amostrais com base na similaridade fisiográfica. as unidades amostrais encontra-se na Figura 5. Observa-se um arranjo similar ao obtido na comparação entre a biodiversidade de cada unidade ambiental considerada, permitindo evidenciar um conjunto de sistemas dotados de pequenas

dimensões gerais e baixa diversificação interna (grupo 1 - rio Macacu em E1 e córregos Colibri, Valona e Esgoto), um complexo de ambientes de grande porte e muito diversificados quando comparados com os demais (grupo 2 - Macacu E2 e E3) e um ambiente em situação intermediária (córrego Valério), neste caso mais fortemente ligado ao grupo 1.

Comparando as matrizes de similaridade alcançadas obteve-se $r=0,734$ sendo $\mathrm{P}=0,05$. Estes valores demonstram que as variações na biodiversidade seguem o mesmo sentido das variações físicas dos ambientes amostrados, logo uma hierarquização de ambientes quanto a sua diversidade física, complexidade e dimensões gerais representa, ao mesmo tempo, uma hierarquia de biodiversidade.

Visto que o número de espécies (S) se comporta como uma função das variáveis adotadas $[\mathrm{S}=f(+$ Área, + Diversidade ambiental, + Extensão do canal principal, + Extensão Total, + Ordem, + Taxa de transformação, + Riqueza de tributários, - Gradiente)] é possível elaborar um índice simples que expresse a importância relativa de cada ambiente dentro do contexto da área de georreferenciamento em estudo. Este índice deve ser concebido dentro do objetivo de permitir uma hierarquização dos ambientes quanto a sua importância no manejo da diversidade biológica da região, podendo ser interpretado como uma medida de sensibilidade ambiental quando assumida a possibilidade de alteração antrópica do ecossistema.

Sendo a condição de maior diversidade biológica a observada no rio Macacu dentro do conjunto amostral E3, os valores das variáveis fisiográficas desta área passam a ser utilizados como elemento relativizador das variáveis mensuradas em cada uma das sete unidades de análise, permitindo expressar a relevância ecológica relativa de cada subsistema ambiental como resultado do somatório dos valores relativos individuais (Vi).

Dentro deste método, sintetizado na Figura 6, o índice de relevância ecológica relativa (IRE) se expressa como IRE $=\Sigma \mathrm{Vi} / \Sigma \mathrm{V}_{\max }$, sendo cada Vi produzido no sentido de gerar 
valores entre 0 e 1 que atuem como uma escala ascendente de relevância. Desta forma os valores máximos relativos de cada variável fisiográfica são iguais a 1. Para o calculo de cada Vi divide-se cada variável medida no ambiente $i$ pelo valor da mesma variável obtida em E3 (sistema com maiores dimensões gerais, logo com maior diversidade biológica).

A única exceção ao processo metodológico supracitado diz respeito ao gradiente (GR) que, por apresentar correlação inversa com a biodiversidade da ictiofauna, é relativizado adotando-se a relação dada pela unidade subtraída pelo resultado da divisão do gradiente observado na sub-bacia em análise $\left(\mathrm{Gr}_{\mathrm{obs}}\right)$ pelo maior gradiente verificado na bacia $\left(\mathrm{Gr}_{\max }\right)$ [

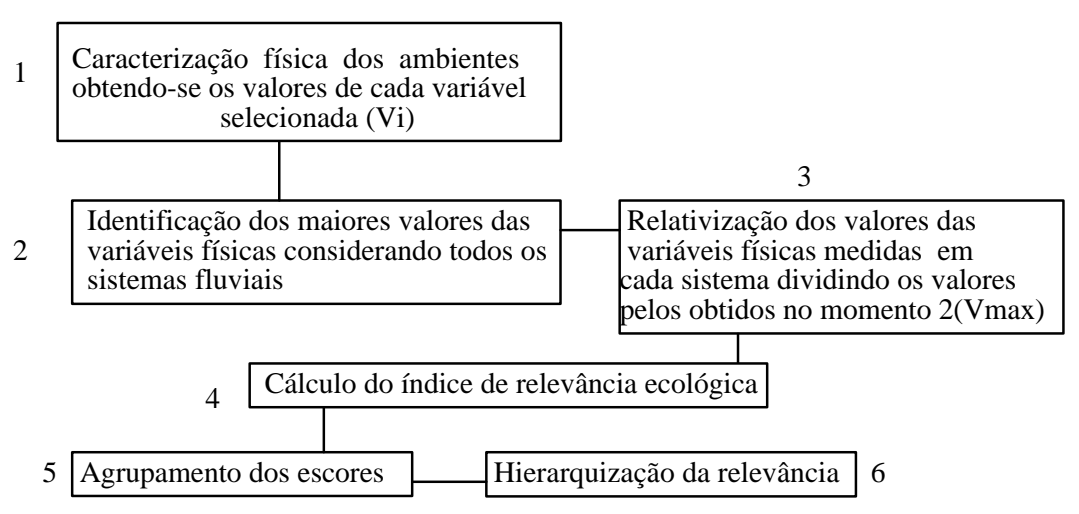

Figura 6 - Síntese das etapas envolvidas no cálculo do índice de relevância ecológica.

$\mathrm{Gr}=1$
$\left.\left(\mathrm{Gr}_{\text {obs }} / \mathrm{Gr}_{\text {max }}\right)\right]$.
O uso do índice de
relevância
ecológica evita
trabalhar com
números muito
elevados gerando
valores entre 0 e 1,
o que em muitas
situações,
especial naquelas

em que o número de ambientes considerado é alto, facilita o processo de visualização dos resultados.

Exemplificando, visto que o ambiente E1 do rio Macacu consiste no seguinte conjunto de características: $\left\{\right.$ área $=2,160 \mathrm{~km}^{2}$, diversidade de ambientes $=0,950$, extensão do canal principal $=2650 \mathrm{~m}$, extensão total $=5150 \mathrm{~m}$, ordem $=2$, número de tributários $=3$, gradiente $=$ $0,17$ e taxa de alteração ambiental $=0,15\}$, que o maior gradiente observado foi de 0,342 (obtido no córrego Esgoto) e que o ponto E3 demarcado no rio Macacu apresenta \{área = $129,25 \mathrm{~km}^{2}$, diversidade de ambientes $=1,813$, extensão do canal principal $=21500 \mathrm{~m}$, extensão total $=201900 \mathrm{~m}$, ordem $=4$, número de tributários $=154$, gradiente $=0,005$ e taxa de alteração ambiental $=0,80\}$ tem-se como valores relativos de E1 \{́́rea $\Rightarrow 2,160 / 129,25=$ 0,01 ; diversidade de ambientes $\Rightarrow 0,950 / 1,813=0,52$; extensão do canal principal $\Rightarrow$ $2650 / 21500=0,12$; extensão total $\Rightarrow 5150 / 201900=0,02$; ordem $\Rightarrow 2 / 4=0,5$; número de tributários $\Rightarrow 3 / 154=0,01$, gradiente $\Rightarrow 1-0,17 / 0,34$; taxa de alteração ambiental $\Rightarrow 0,15 / 0,8$ 
$=0,18\}$. Logo, IRE Macacu E1 $=(0,01+0,52+0,12+0,02+0,5+0,01+0,5+0,18) / 8 \therefore$ IRE Macacu E1 = 0,23

Este processo, quando aplicado aos demais ambientes em estudo, gera os seguintes escores: Macacu E1 = 0,23; Macacu E2 = 0,69, Macacu E3 = 0,98, córrego Valona = 0,29, córrego Colibri $=0,31$, córrego Valério $=0,42$. Comparando as matrizes de semelhança de biodiversidade com a similaridade do índice ambiental obtem-se $r=0,813$ sendo $P=0,002$ (ver agrupamento na Figura 7) mostrando, consequentemente, que o índice proposto, por trabalhar dentro de uma escala previamente elaborada para representar a relevância dos ambientes fluviais gera escores mais fortemente associados à real diversidade biológica dos sistema estudados que os dados brutos.

Verifica-se, portanto, que a aplicação do índice proposto permite identificar, através de

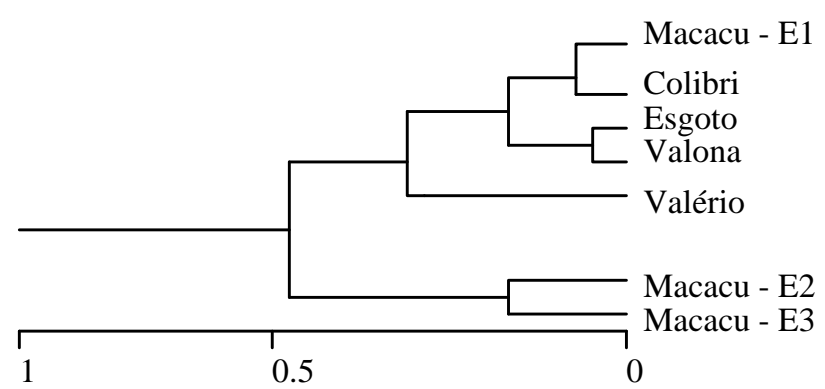

Figura 7 - Agrupamento das unidades amostrais com base nos valores do índice de relevância ecológica. um critério lógico e não subjetivo, áreas prioritárias para a conservação da diversidade biológica da ictiofauna de sistemas fluviais, em um processo de baixo custo e plenamente compatível com a disponibilidade de tempo de estudos de avaliação ambiental.

Para a aplicação deste método ressalta-se a necessidade de serem consideradas unidades naturais, ou seja, que representem um único compartimento do conjunto de ecossistemas que integram um ambiente fluvial (SCHAFER, 1985). No presente estudo, a ausência de extinções ao longo do gradiente hidrodinâmico e, consequentemente, a inexistência de espécies endêmicas do curso superior, ilustra que o alto curso do rio Macacu é um ecossistema bem definido. Em caso de comparações entre bacias isoladas as mesmas devem ser conduzidas apenas em conjuntos monofiléticos de sistemas (i.e., que possuam uma história biogeográfica comum).

Logicamente, os resultados aqui apresentados devem ser encarados como uma primeira aproximação que demanda a realização de novos estudos com a metodologia proposta enfocando sistemas fluviais com características gerais distintas das observadas no alto curso do rio Macacu. 


\section{REFERÊNCIAS BIBLIOGRÁFICAS}

ARGENTO, M.A. Caracterização morfométrica do alto rio Macacu. Uma abordagem quantitativa. Anuário do Instituto de Geociências, v. 1985. p.42-71, 1985.

BIZERRIL, C.R.S.F. Análise taxonômica e biogeográfica da ictiofauna de água doce do leste brasileiro. Acta Biol. Leopoldensia, v. 16, n.1, p. 51-80, 1994.

BIZERRIL, C.R.S.F. Análise da distribuição espacial da ictiofauna de uma bacia do leste brasileiro. Arquivos de Biologia e Tecnologia, v. 38, n. 2, p. 477-500, 1995.

BRITO, E.J.G.N. Estudos de Impacto Ambiental (EIA) e Relatório de Impacto Ambiental (RIMA): erros e acertos. In: TAUK-TORNISIELO, S.M., GOBBI, N., FORESTI, C. \& LIMA, S.T. Análise ambiental - Estratégias e ações. São Paulo: Fundação Salim Farah Maluf, 1995. P. 255-259.

BURGESS, W.E. An atlas of freshwater and marine catfishes - A preliminary survey of the Siluriformes. New Jersey: T.F.H. Publications, 1989. p. 784.

CHRISTOFOLETTI, A. Análise morfométrica de bacias hidrográficas. Not. Geomorf., v. 9, n. 18, p. 3564, 1969.

CHRISTOFOLETTI, A., GIRARDI, L.H.O. \& TAVARES, A.C. Análise comparativa e classificatória de bacias hidrográficas paulistas. Geomorfologia, v. 45, p. 1-9, 1974.

COLINVAUX, P. Ecology. New York, Jonh Wiley \& Sons, 1986. p.725.

CUNHA, S.B. \& GUERRA, A.J.T. Geomorfologia: Exercícios, técnicas e aplicações. Rio de Janeiro: Ed. bertrand Brasil, 1996. p. 345.

DIAMNOD, J.M \& MAY, R.M. Island biogeography and the design of natural reserves. In: MAY, R.M., Theoretical ecology: Principles and applications. Philadelphia: Sauders, 1976. p. 163-186.

FEEMA Diagnóstico ambiental do Estado do Rio de Janeiro. Rio de Janeiro: FEEMA, 1979. p 103.

JENNINGS, M.D \& REGANOLDS, J.P. A theoretical basis for managing environmentally sensitive areas. Environmenntal Conservation, v. 18, n. 3, p. 211-218, 1991.

LAMEGO, A.R. O Homem e o brejo. Rio de Janeiro: Ed Lidador, 1945. p. 217.

LUDWIG, J.A. \& REYNOLDS, J.F. Statistical ecology - A primer on methods and computing. New York: Jonh Wiley \& Sons, 1985. 337 pp.

MacCARTHUR, R.H. \& WILSON, E.O. An equilibrium theory on insular zoogeography. Evolution, v. 5, n. 17, p. 373-387, 1963.

MacCARTHUR, R.H. \& WILSON, E.O. The theory of island biogeography. Princeton: Pinceton University Press, 1967. p. 275.

MANTEL, N. The detection of disease clustering and a generalized regression approach. Cancer Research, v. 27, p. 209-220, 1967.

PENCZAK, T \& R.H.K. MANN The impact of stream order on fish populations in the Pilica drainage basin, Poland. Polskie Archiwum Hydrobioligii, v. 37, n. 1-2, p. 243-261, 1990.

RICKLEFS, R.E. \& D. SCHLUTER Species diversity in ecological communities. Chicago: University of Chicago Press, 1993. p. 414.

SCHAFER, A. Fundamentos de ecologia e biogeografia das águas continentais. Porto Alegre: Editora da UFRGS, 1985. p. 532 .

SHANNON, C.E. A mathematical theory of communication. Bell System Tech. J., v. 27, p. 379-423, 1948.

SHELFORD, V. Ecological sucession. I. Stream fishes and the method of physiografic analysis. Biol. Bull., v. 21, p. 9-35, 1911.

SIMBERLOF, D. An equilibrium theory of biogeography and ecology. An. Rev. Ecol. Systematics, v. 5, p. 161-182, 1974.

SIMBERLOF, D. \& WILSON, E.O. Experimental zoogeography of islands: The colonization of empty islands. Ecology, v. 50, p. 278-296, 1969.

SIMBERLOF, D. \& WILSON, E.O. Experimental zoogeography of islands. A two-year record of colonization. Ecology, v. 51, p. 934-937, 1970. 
SNEATH, P.H.A. \& SOKAL, R.R. Numerical taxonomy. San Francisco: Freeman, 1973. p. 571.

STRAHLER, A.N. Hypsometric (area-latitude) analysis of erosional topography. Geol. Soc.America Bulletin, v. 63, p. 1117-1142, 1952.

WHITESIDE, B.G. \& McNATT, R. Fish species diversity in relation to stream order and physicochemical conditions in the Plum Creek drainage basin. Amer. Mid. Nat., v. 88, n. 1, p. 90101, 1972.

ZAR, J.H. Biostatistical analysis. New Jersey: Prentice.Hall, 1984. p. 718.

Received: 01 April 1997; Revised: 16 May 1997;

Accepted: 08 June 1998. 